\title{
Z Cam stars: A particular response to a general phenomenon
}

\author{
V. Buat-Ménard ${ }^{1}$, J.-M. Hameury ${ }^{1}$, and J.-P. Lasota ${ }^{2}$ \\ 1 Observatoire de Strasbourg, UMR 7550 du CNRS, 11 rue de l'Université, 67000 Strasbourg, France \\ e-mail: hameury@astro.u-strasbg.fr \\ 2 Institut d'Astrophysique de Paris, 98bis boulevard Arago, 75014 Paris, France \\ e-mail: lasota@iap.fr
}

Received 11 October 2000 / Accepted 22 January 2001

\begin{abstract}
We show that the disc instability model can reproduce all the observed properties of Z Cam stars if the energy equation includes heating of the outer disc by the mass-transfer stream impact and by tidal torques and if the mass-transfer rate from the secondary varies by about $30 \%$ around the value critical for stability. In particular the magnitude difference between outburst maxima and standstills corresponds to observations, all outbursts are of the inside-out type and can be divided into two classes: long (wide) and short (narrow) outbursts, as observed. Mass transfer rate fluctuations should occur in other dwarf novae but one can exclude variations similar to those observed in magnetic systems (AM Her's and some DQ Her's) and some nova-like systems (VY Scl's), in which $\dot{M}$ become very small during low states; these would produce mini-outbursts which, although detectable, have never been observed.
\end{abstract}

Key words. accretion, accretion discs - instabilities - (stars:) novae, cataclysmic variables - (stars:) binaries: close

\section{Introduction}

Dwarf novae are a subclass of cataclysmic variable stars, which are close binary systems in which matter transferred from a Roche-lobe filling secondary star is accreted by a primary white dwarf (see Warner 1995). In dwarf novae accretion proceeds through a disc which is the site of more or less regular outbursts. The recurrence time of these 2-6 mag outbursts can range from 10 days to several tens of years. The dwarf nova outbursts are attributed to a thermal-viscous instability occurring in the accretion disc. Although the thermal-viscous disc instability model (DIM) identifies a physical cause for the instability, partial hydrogen ionization, the outburst cycles it produces differ in many important respects from the observed ones (see e.g. Smak 2000; Lasota 2000). It is now rather widely accepted that the original DIM has to be completed by various physical processes such as irradiation of the disc and the secondary star, inner disc truncation and mass transfer fluctuations. Also heating of the disc by the mass-transfer stream impact and by tidal torque dissipation plays an important role in the dwarf-nova outburst cycle (BuatMénard et al. 2001, hereafter Paper I).

Dwarf novae can be divided into three subclasses: U Gem-type stars which have the most regular outburst

Send offprint requests to: V. Buat-Ménard, e-mail: buat@astro.u-strasbg.fr cycles; SU UMa-type stars showing both short and very long outbursts (superoutbursts) and Z Cam stars. The last group is characterized by a "standstill" phenomenon: the decline from outburst maximum is interrupted and the luminosity of the systems settles to a value $\sim 0.7$ mag lower than the peak luminosity. In some cases the magnitude difference is smaller (see e.g. Fig. 19 in Lin et al. 1985). Such standstills may last from ten days to years. After that, the system luminosity declines to the usual quiescent state. In his pioneering work, Osaki (1974) interpreted standstills as stable phases of accretion in the framework of the disc instability model he then proposed. In his discradius and mass-transfer rate diagram, Smak (1983) described these stars as an intermediate case between stable nova-like stars and unstable dwarf novae. Meyer \& MeyerHofmeister (1983) proposed that Z Cam stars are dwarf novae with a mass-transfer rate that fluctuates about the critical rate. Lin et al. (1985) concluded that moderate fluctuation of the mass-transfer rate can produce Z Camtype light curves. This is now widely accepted, but until now no model has been able to reproduce the observed light curves of Z Cam (e.g. King \& Cannizzo 1998).

We applied the disc instability model to a system in which the mass transfer rate from the secondary star $\dot{M}_{2}$ is assumed to vary around the critical rate $\dot{M}_{\mathrm{c}}$, above which the disc is stable. In Sect. 2 we show that the magnitude difference between standstills and outburst peaks 
depends on the difference between $\dot{M}_{\mathrm{c}}$ and the actual mass transfer rate reached during standstill $\dot{M}_{\mathrm{h}}$. We also show that it is easier to find a large enough $\Delta_{\text {mag }}$ when the model includes the outer disc heating by the stream impact and the tidal dissipation as discussed in Paper I. In addition, including these additional heating processes in the DIM allows to obtain inside-out outbursts as observed in some Z Cam stars. We show therefore that in such a generalized DIM the response of a dwarf nova disc to lowamplitude mass-transfer rate variations is the explanation of the Z Cam phenomenon.

Mass-transfer rate variations are also occurring in other dwarf novae (e.g. Smak 1999, 2000; Hameury et al. 2000). The brightening of the hot spot, the region where the mass-transfer stream interacts with the outer disc's rim, which is observed near the outburst maxima, should be the result of a mass-transfer rate enhancement. Large variations of the mass transfer rate are directly observed in polars, which are magnetic cataclysmic variables with no accretion discs. The prototype, AM Her shows large amplitude variations of the mass transfer rate which can be vanishingly small during low states. Similar luminosity drops observed in some DQ Her stars, magnetic nonsynchronous binaries and in VY Scl, nova-like systems with orbital periods between 3 and 4 hours, are also attributed to mass-transfer fluctuations. However, because of the presence of a disc, it is much more difficult to convert these luminosity fluctuations into mass-transfer rate variations. Contrary to Schreiber et al. (2000), who used a code in which the outer boundary radius is kept fixed, we find using the correct outer boundary condition, that such drastic mass-transfer fluctuations do not occur in most dwarf novae. King \& Cannizzo (1998) reached the same conclusion as ours using a fixed outer radius and varying the mass-transfer rate by 6 orders of magnitude.

\section{Modeling the Z Cam phenomenon}

\subsection{Model and parameters}

We use the version of the disc instability model described in Hameury et al. (1998). We assume that $\alpha=\alpha_{\text {cold }}=$ 0.04 in quiescence and $\alpha=\alpha_{\text {hot }}=0.2$ in outburst. As in Paper I the fixed, inner radius is at $r_{\text {in }}=10^{9} \mathrm{~cm}$. We allow for the inclusion of the stream impact and tidal dissipation effects (see Paper I for details). We do not include the effects of the secondary star irradiation, i.e. the mass-transfer rate variations are supposed, in this model, to be unrelated to the accretion onto the white dwarf.

We choose Z Cam as the system to model. From the Ritter and Kolb's catalogue (1998) we took mass of the primary $M_{1}=1 M_{\odot}$, the mass of the secondary $M_{2}=$ $0.7 M_{\odot}$ and the orbital period $P_{\text {orb }}=6.96 \mathrm{hr}$. We use the tables from Lubow \& Shu (1975) and Paczyński (1977) to estimate the circularization radius $r_{\text {circ }}=1.5210^{10} \mathrm{~cm}$ and the mean outer disc radius $\left\langle r_{\text {out }}\right\rangle=5.410^{10} \mathrm{~cm}$. The size of the disc is controlled by the tidal torques which depend on the value of the circularization radius (see Hameury et al. 1998 and Paper I for details).

We assume that the mass-transfer rate from the secondary varies from a low state $\dot{M}_{1}=<\dot{M}>-\Delta \dot{M}$ to a high state $\dot{M}_{\mathrm{h}}=\left\langle\dot{M}>+\Delta \dot{M}\right.$ with a period $P_{\mathrm{m}}$, where $<\dot{M}>$ is the average mass-transfer rate from the secondary. Transitions between the two states are made by sinusoidal interpolation during a time interval $\Delta t_{\text {trans }}$. In this paper we use $P_{\mathrm{m}}=400$ days; this is long enough to account for relaxation during standstills and to allow several outbursts cycles. The transition time is taken to be $\Delta t_{\text {trans }}=20$ days.

The brightening of the quiescent level and the outburst amplitude reduction observed in some Z Cam systems (Szkody \& Mattei 1984) may result from a particular time-dependence of the mass-transfer rate fluctuations but we are not attempting to model such effects here. The $\dot{M}$ variations used in this paper are not supposed to correspond to any particular physical mechanism. We are interested in the disc's response to mass-transfer rate fluctuations and not in the possible causes of these variations. The latter are far from being known. Irradiation induced cycles of mass-transfer rate variation are excluded in this context as they last much longer than the observed interval between standstills (Gontikakis \& Hameury 1993). Star spots could be be responsible for $\dot{M}$ fluctuations that are relevant for Z Cam stars (Livio \& Pringle 1994; King \& Cannizzo 1998).

\subsection{Mass-transfer fluctuations}

Figure 1 shows the results of our calculations when no additional disc heating is included. We assumed the average mass-transfer rates $\langle\dot{M}>$ to be equal to the critical rate $\dot{M}_{\mathrm{c}}=810^{17} \mathrm{~g} \mathrm{~s}^{-1}$ (see Hameury et al. 1998 for the formula). We took $\Delta \dot{M} /<\dot{M}>=30 \%$. The modulation of the mass-transfer rate occurs at $t=200+k P_{\mathrm{m}}$ day. As can be seen, the outburst amplitudes gradually increase after a standstill, as the disk relaxes towards the new situation in which the mass transfer rate is reduced. Since now mass accumulates in the disc, it is more massive than before, when it was steady. It is also larger after an initial contraction phase due to the passage of a cooling front. All outbursts are of the outside-in type, as the mass transfer rate is large (see Paper I). The instability sets in at approximately the same position $\left(\sim 310^{10} \mathrm{~cm}\right.$, except for the last outburst which is triggered by an increase of the mass transfer rate from the secondary). As only a small fraction of the disk mass is accreted during outbursts, a relaxed regime is reached only after a large number of outbursts, close to the end of the dwarf nova phase. The luminosity increase of successive outbursts, a characteristic feature of this model, is not observed in reality. To solve this problem one could use severals remedies. For example, one could add to the model mass-transfer rate enhancements due to the irradiation of the secondary by the accretion flow (Smak 1999; Hameury et al. 2000). We will show 


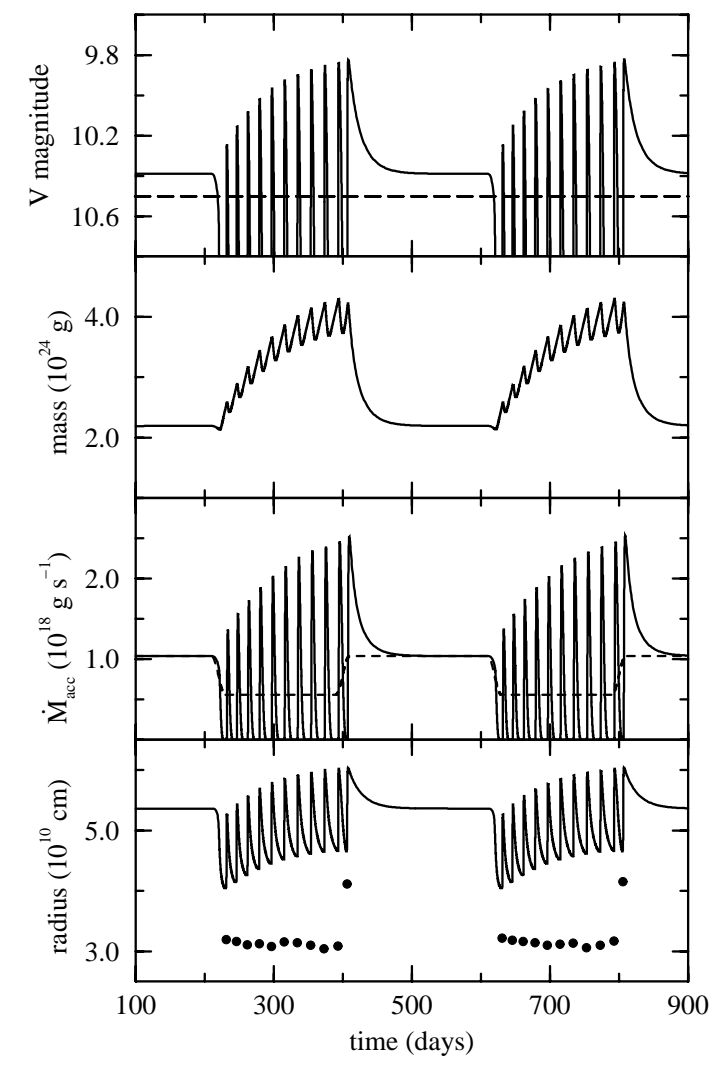

Fig. 1. Results of the standard DIM for the parameters of $\mathrm{Z}$ Cam, assuming a modulated mass-transfer rate $8.010^{17} \pm$ $30 \% \mathrm{~g} \mathrm{~s}^{-1}$. The upper panel shows the visual magnitude (solid line). The dashed line represents the $V$ magnitude (10.5) of a disc which accretes at exactly the critical value: $\dot{M}_{\mathrm{c}}=$ $8.010^{17} \mathrm{~g} \mathrm{~s}^{-1}$. The second panel from above shows variations of the disc's mass. The third one represents the mass accretion rate onto the white dwarf (solid line), as well as the mass transfer rate from the secondary (dashed line). The bottom panel shows the variations of the outer disc radius. The dots show point where the instability is triggered at the onset of an outburst. All outbursts are of the outside-in type

below, however, that this is not necessary since taking into account the heating by the stream impact and/or tidal torque dissipation (Paper I) gives very satisfactory results. In any case Fig. 1 shows that $\Delta_{\text {mag }}<0.6 \mathrm{mag}$, which is too low since the observed magnitude difference often exceeds $0.7 \mathrm{mag}$. To get a better result would require the use of some fine tuning. For obvious reasons, the standstill luminosity cannot be lower than that corresponding to $\dot{M}_{\mathrm{c}}$, i.e. for our parameters the brightest standstill magnitude can only be $m_{V}=10.5$. The outburst peak luminosity depends mainly on the disc radius, which only weakly depends on the mass-transfer rate. Therefore the magnitude at maximum, in our case $m_{V}=9.8$ is just an invariant of the model. Therefore to obtain the observed $\Delta_{\text {mag }} \sim 0.7$ would require $\dot{M}_{\mathrm{h}} \simeq \dot{M}_{\mathrm{c}}$. Fine tuning would be required for the $\sim 30 \mathrm{Z}$ Cam known, because the critical and the peak accretion rate have the same $r$-dependence.

Another problem with our parameters is that the required vicinity to the critical rate implies very high mass- transfer rates. This produces outside-in outbursts in contradiction to observation. Moreover, according to the most recent work on CV's evolution Z Cam should have a masstransfer rate lower than $\sim 3.210^{17} \mathrm{~g} \mathrm{~s}^{-1}$ (Baraffe \& Kolb 2000).

It is therefore possible to produce a $\mathrm{Z}$ Cam-type light curve by varying the mass-transfer rate around the value critical for disc stability, but neither the parameters of this light-curve nor the required parameters of the systems are satisfactory. One should also note that the range of possible parameters is very restricted. While the mass-transfer rate is $810^{17} \mathrm{~g} \mathrm{~s}^{-1}$ the accretion rate at maximum is only less than a factor 2 to 3 larger $\left(1.3-2.510^{18} \mathrm{~g} \mathrm{~s}^{-1}\right)$. This means that a mass-transfer rate enhancement by a factor more than $\sim 2$ would produce a "Z Cam" light-curve with $\Delta_{\text {mag }}<0$ as in King \& Cannizzo (1998), who increased the mass-transfer rate by a factor 6 . The reason for this very narrow range of mass-transfer fluctuations which allow a Z Cam-type standstill is that close to the disc's outer edge non-local effects during the outburst bring the surface density very close to the critical value $\Sigma_{\min }$ (see e.g. Smak 1998, Fig. 4), i.e. $\dot{M}$ close to $\dot{M}_{\mathrm{c}}$. Since at maximum the accretion rate in the disc is roughly constant $\dot{M}_{\max } \sim \dot{M}_{\mathrm{c}}$. Esin et al. (2000) assumed that $\dot{M}_{\max }=\dot{M}_{\mathrm{c}}$ and deduced that an enhancement of mass transfer rate above $\dot{M}_{\mathrm{c}}$ will always produce a standstill brighter than the outburst maximum. This is not true because in reality the peak accretion rate is larger than $\dot{M}_{\mathrm{c}}$ by a factor of about 2. Therefore King \& Cannizzo (1998) guessed correctly that they had increased the mass-transfer rate by an amount too large to get a Z Cam-type standstill. One should note, however, that some low-mass X-ray binary transient systems show plateau brighter than a preceding local maximum which could be due mass-transfer enhancements by factors larger than 2 (see e.g. Esin et al. 2000).

\subsection{Effects of heating by stream impact and tidal torque dissipation}

We found in the preceding section that in order to produce credible Z Cam light-curves one should lower the critical mass-transfer rate. One needs therefore a physical mechanism which would produce such an effect without lowering the peak luminosity. Heating of the disc by the impact of the mass-transfer stream from the secondary and/or by tidal torque dissipation (Paper I) could do the job: it has a stabilizing effect, therefore allowing the disc to be dimmer during standstills, but does not affect the outbursts properties near the dwarf-nova maximum because (i) as mentioned earlier they depend only weakly upon the average mass transfer rate, and (ii) the additional heating is small compared to the energy released during outbursts. Moreover, heating of the outer disc reduces both the value of the critical mass-transfer rate $\dot{M}_{\mathrm{c}}$ and the value $\dot{M}_{\mathrm{AB}}$ for which the outburst type changes; this makes outside-in outbursts possible for reasonably low values of the mass transfer rate (Paper I). 
The relative difference $\left(\dot{M}_{\mathrm{c}}-\dot{M}_{\mathrm{AB}}\right) / \dot{M}_{\mathrm{c}}$ is also slightly reduced. It is thus possible to have also inside-out outbursts during the unstable phase of Z Cam systems even for moderate fluctuations of the mass-transfer rate. This could explain why type B outbursts are observed in some Z Cam systems, while other would rather have type A ones (Warner 1995).

In a recent paper, Stehle \& King (2000) argued that heating of the disc by the stream impact is responsible for the standstill luminosity being less than the outburst peak luminosity, provided that the amplitude of the mass transfer fluctuations is large enough: they require that the mass transfer is low enough during the unstable phase for heating by the stream impact to be negligible, and large during standstill. However, as clearly showed by our model calculations such constraints are not necessary. In addition, Stehle \& King model would require very fine tuning: the mass transfer fluctuations would have to be large enough for heating by the stream impact to be, depending on the mass-transfer fluctuation phase, either negligible or quite important, and yet the mass transfer rate during standstills could not be larger than approximately (1.5-2) $\dot{M}_{\mathrm{c}}$, in order to prevent the standstill phase from being too bright. Obviously one would expect to find systems in which both conditions are not satisfied and such systems would have excessively bright standstills.

Including only the heating of the disc by the stream impact (see Paper I) gives $0.55<\Delta_{\mathrm{mag}}<1.00 \mathrm{mag}$ for a fluctuation of $30 \%$ around $\langle\dot{M}\rangle=3.010^{17} \mathrm{~g} \mathrm{~s}^{-1}$. The values of both the mass transfer rate and the magnitude difference are thus in a better agreement with the observed properties of Z Cam stars. Adding to this effect the tidal dissipation term in the energy balance equation, gives $0.6<\Delta_{\mathrm{mag}}<0.8 \mathrm{mag}$ for a fluctuation of $30 \%$ around $\langle\dot{M}\rangle=2.010^{17} \mathrm{~g} \mathrm{~s}^{-1}$ (Fig. 2), which is very satisfactory. The cause of this success is a faster relaxation of the disc after the drop in the mass-transfer rate, when both effects are included. This is due to two effects. First, a larger mass fraction is accreted during an outburst: $\Delta M / M \sim 0.25$. Second, the difference between the disc mass during standstill and during the outburst phase is now smaller: additional heating reduces the difference $\Sigma_{\max }-\Sigma_{\min }$ in the outer regions of the disc (Paper I), where most of its mass sits. In addition, for mass-transfer rates $\left\langle\dot{M}>\sim 2.010^{17} \mathrm{~g} \mathrm{~s}^{-1}\right.$ the two external heating mechanisms produce narrow and wide outbursts (see Paper I for details). Such outbursts are observed but cannot be reproduced by the standard DIM (Smak 2000). Our longer outbursts correspond to the "plateau" outbursts of Oppenheimer et al. (1998).

In this particular model all outbursts inside-out type. In Z Cam and AH Her, outbursts are presumed to be of type $\mathrm{B}$ whereas in RX And they are presumed to be of type A (see Table 3.6 in Warner 1995). As mentioned above, with the inclusion of heating by stream impact and tidal torques, $\left(\dot{M}_{\mathrm{c}}-\dot{M}_{\mathrm{AB}}\right) / \dot{M}_{\mathrm{c}}$ is reduced so that it is possible to obtain type $\mathrm{B}$ outbursts even with a moderate mass

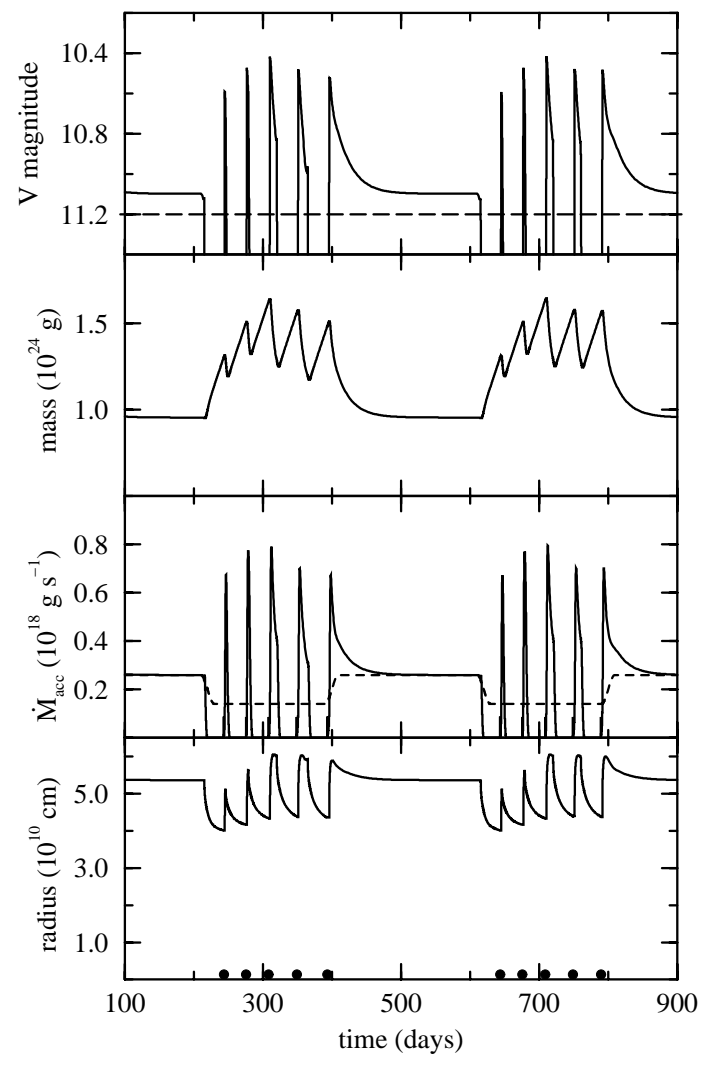

Fig. 2. Light curve obtained for Z Cam parameters with the DIM including stream impact and tidal torques effects. $\dot{M}_{2}=2.010^{17} \pm 30 \% \mathrm{~g} \mathrm{~s}^{-1}$. The dashed line shows the standstill magnitude (11.2) for the critical mass transfer rate $\dot{M}_{\mathrm{c}}=2.410^{17} \mathrm{~g} \mathrm{~s}^{-1}$

transfer rate fluctuation. Depending on the characteristics of these fluctuations, one could obtain either type A outbursts, or type B ones, or a mixture of both. In addition, the last outburst of the unstable phase should be of type A if $\dot{M}>\dot{M}_{\mathrm{AB}}$ before it starts. If the mass transfer rate varies smoothly, one would therefore expect to observe type A outbursts just before a standstill; this would not be the case if $\dot{M}$ varied rapidly (for example if the secondary irradiation during outbursts affects $\dot{M})$.

As $\dot{M}_{\mathrm{c}}$ is close to $\dot{M}_{\mathrm{AB}}\left(\dot{M}_{\mathrm{c}} \sim 1.2 \dot{M}_{\mathrm{AB}}\right.$ for $\mathrm{Z}$ Cam and SS Cyg), one should expect U Gem systems in which outside-in outbursts occur, to have standstills from time to time. Warner (1995) noted that the suggestion that "all U Gem stars are unrecognized Z Cam stars" is somewhat exagerated, but a number of U Gem stars with short recurrence times have been reclassified as Z Cam stars. It would therefore not be surprising if SS Cyg were to exhibit a standstill, even though none has been observed in the past 100 years. This only requires a slight increase of the mean mass transfer rate.

In general, despite the simplicity of its assumptions, our model represents very well the outburst properties of Z Cam stars. The recurrence time is 32 days between narrow outbursts and 40 days between plateau outbursts, the duration of the two narrow outbursts 8 and 9 days, of 


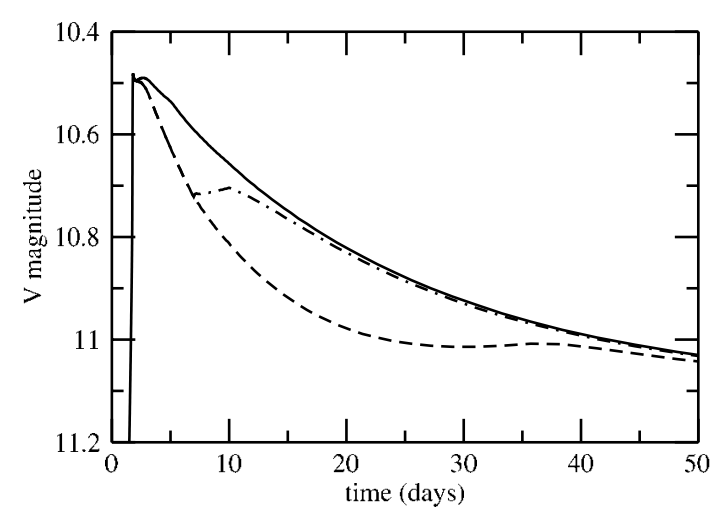

Fig. 3. Last outburst before standstill. The solid line is an enlargement of Fig. 2, for which mass transfer increases early in the outburst; the dot-dashed curve is obtained when mass transfer starts increasing linearly 5 days after the outburst peak, and reached the standstill value 3 days later; the dashed curved corresponds to a case where the increase in $\dot{M}$ also begins 5 days after the outburst maximum, but with a timescale of 30 days. The latter curve requires some tuning

the two "plateau" outbursts 16 and 20 days. These results compare reasonably well with the observed values: the average cycle length of common and plateau outbursts is 23 and 31 days respectively, and their average duration 10 and 17 days. However, the duration of the last outburst before standstill is too long compared to observations: we predict that it should be significantly longer than the previous ones, whereas this is not the case for the observed outbursts. As a matter of fact, the transition between the outburst phase and standstill depends significantly on how $\dot{M}$ increases before standstill. Figure 3 shows different transitions to standstill, for the same parameters as in Fig. 2, except that mass transfer now increases during the outburst. As can be seen, shorter outbursts are easily obtained. This might be used as an indication that $\dot{M}$ increases a few days after the beginning of an outburst, maybe as the result of illumination; one must however keep in mind that this is a relatively minor detail compared to many uncertainties of the physics of the model (viscosity prescription, etc.).

Z Cam-type light curves can therefore be very well reproduced by the disc response to a simple mass-transfer rate fluctuations if additional heating is included.

\section{3. $\dot{M}_{2}$ variation in other dwarf novae}

Variations of the mass transfer rate from the secondary are not restricted to the $\mathrm{Z}$ Cam subclass of dwarf novae. The variety of outburst types observed in most dwarf-nova light curves implies the existence of a free parameter for any given system, and the mass transfer rate from the secondary is a good candidate; as discussed in Paper I the main different outburst types (outside-in, inside-out, narrow and wide outbursts) are triggered for different mass transfer rates. Finally, the large luminosity variations of AM Her systems, CVs with no accretion discs, must di-

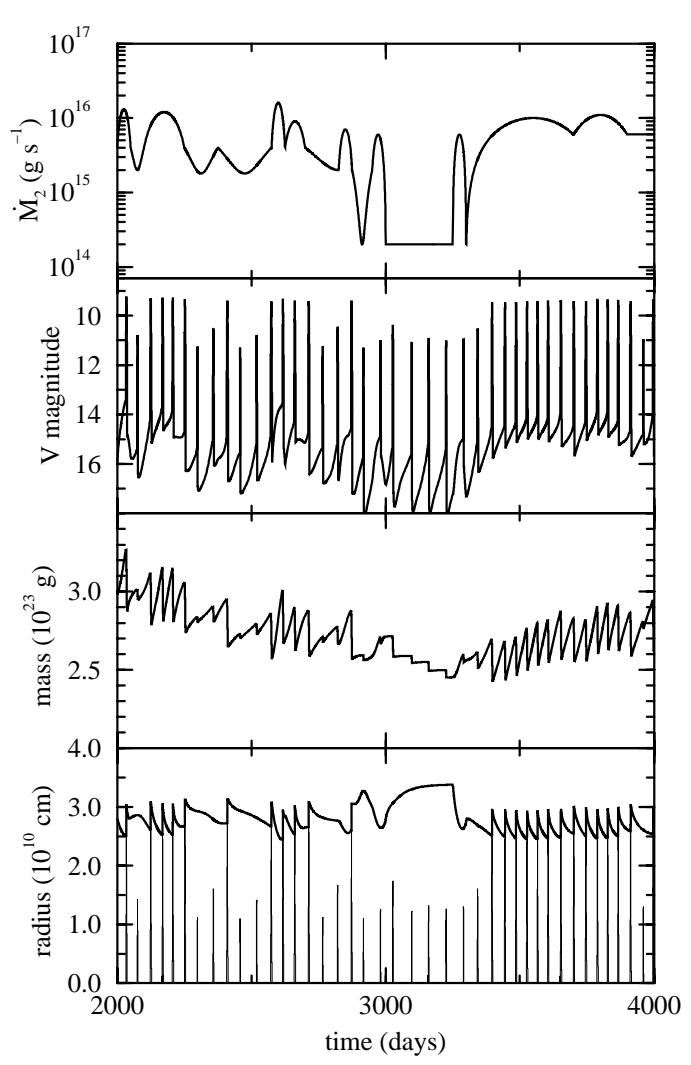

Fig. 4. DIM results for a fictitious dwarf nova with the parameters and the mass-transfer rate variation (top panel) of AM Her. The top panel gives the mass transfer rate from the secondary, the second panel the disc visual magnitude, the third panel its mass, and the bottom panel the outer disc radius (thick solid line), and the position of the external heating or cooling front (thin solid line)

rectly result from fluctuations of the mass transfer rate from the secondary. Also in other systems, the magnetic non-synchronous DQ Her stars and nova-like VY Scl stars, the large brightness variations observed are attributed to mass-transfer fluctuations (see Garnavich \& Szkody 1988 and references therein).

However, the reasons for these fluctuations are not really known; irradiation of the donor star and star spots have been invoked as possible causes for the variations, but no reliable model has been yet proposed. Moreover, the amplitude of these fluctuations remains, in the general case, very uncertain. Smak (1995) studied the luminosity of the hot spot in Z Cha and U Gem and showed that during outburst the mass transfer rate can increase by a factor of about 2; there was however no indication of very significant long term variations. We have shown that for Z Cam system a mass-transfer rate variation of less than $50 \%$ (i.e. a factor less than 2) is required to explain their light curves. On the other hand, Schreiber et al. (2000) suggested that mass transfer rate variations similar to those observed in AM Her could be present in dwarf novae systems. In such a case, the mass transfer rate $\dot{M}$ could vary by one order of magnitude and 
become vanishingly small during low states. This behaviour was observed in synchronous magnetic systems, and could be quite general; one must however point out again that there has been no report of disappearance of the hot spot in dwarf novae, even if this might be due to insufficient statistics. Two dwarf novae were observed in a low state during quiescence: HT Cas and BZ UMa. BZ UMa was found to be at $17.8 \mathrm{mag}$ instead of the usual 16 mag (Kałużny 1986). BZ UMa, however, could be a DQ Her star (Kato 1999). In the 5 year of RoboScope observations, during which HT Cas was in quiescence, long term (days to hundreds of days) $\sim 1.8 \mathrm{mag}$ variations were observed. The quiescent luminosity varied from about 15.9 to $17.7 \mathrm{mag}$ (Robertson \& Honeycutt 1996). During one of the low states Wood et al. (1995) observed eclipsed $\mathrm{X}$-ray emission which shows that accretion onto the white dwarf was still going on. Although HT Cas is an eclipsing system it is notorious for not showing the presence of a hot spot, so there is no direct evidence that the low states are due to a mass-transfer fluctuation of a corresponding size. The dwarf nova WW Ceti shows 1 mag variations during quiescence, which could be due to masstransfer fluctuations (Ringwald et al. 1996). RoboScope observations of eight well sampled SU UMa stars show no evidence for large quiescent variations. The evidence for large mass-transfer fluctuations in dwarf novae is therefore rather sparse. In any case, if the observed variations are due to mass-transfer variations, they are smaller than nova-like (or AM Her) variations: 1 to 1.8 mag against 2 to 6 mag. This is consistent with the conclusion that in dwarf novae such huge variations can be excluded by the DIM, as we will show below.

Schreiber et al. (2000) claim that although the cessation of mass transfer has immediate consequences on the light curves, these are limited to shortening the outburst duration and increasing the recurrence time. This is apparently in contradiction with the findings of King \& Cannizzo (1998) that the cessation of mass transfer results in a 2 mag decrease of the outburst amplitude; it must however be noted that the mass-transfer rate during low state was reduced by 6 orders of magnitude in King \& Cannizzo's models, but only by one order of magnitude in Schreiber et al. A major drawback of both sets of calculations, however, is the use of a fixed outer disc radius as a boundary condition; this severely affects the light curves, and is responsible for the occurrence of mini-outbursts at a constant $\dot{M}$ (Hameury et al. 1998).

To see what are the real predictions of the DIM we applied therefore our disc instability model to a fictitious dwarf nova in which the mass-transfer rate variations are similar to those of AM Her (see Fig. 4). We include stream impact and tidal dissipation for a dwarf nova with $M_{1}=0.6 M_{\odot}, M_{2}=0.26 M_{\odot}, P_{\text {orb }}=3.08 \mathrm{hr}$ and $r_{\text {out }}=2.710^{10} \mathrm{~cm}$. The mass-transfer rate varies from 0.01 up to $410^{16} \mathrm{~g} \mathrm{~s}^{-1}$; the variations are similar to those in Schreiber et al. (2000).

Three different types of modulations can be distinguished in the mass transfer curve: during the initial phase, $\dot{M}$ varies by a factor $\sim 10$; then in some phases the mass transfer almost completely ceases, being reduced by a factor 100, during either a short or a long time; during the last 700 days, only small variations are present. When the mass transfer rate remains larger than a few $10^{15} \mathrm{~g} \mathrm{~s}^{-1}$, the calculated light curve shows little variations; the outburst peak magnitudes do not change by more than $0.2 \mathrm{mag}$ and of the recurrence time remains the same, to within a few day. When the mass transfer rate increases, so does the outburst peak magnitude with a delay of about ten days. By contrast, during the low state only mini-outbursts, fainter by 2 mag than the normal ones, are present. The recurrence time increases by up to a factor 2 .

This is quite similar to the findings of King \& Cannizzo (1998) - but our mass transfer rate is 4 orders of magnitude larger than theirs, and contradicts Schreiber et al. who consider the same variations of $\dot{M}$ as we do. The reason for such a discrepancy is the difference in the boundary conditions. During an outburst, the disc expands, so that tidal torques can carry the large amount of angular momentum of matter accreted onto the white dwarf. Under the effect of both the tidal torques and the mass transfered from the secondary, the disc contracts during quiescence. When the mass transfer ceases, this contraction does no longer occur, and the disc remains extended, and the surface density is low. The outbursts are still of the inside-out type, but the heating front can no longer propagate into the outer regions where the disc surface density has been reduced below $\Sigma_{\text {min }}$ under the effect of the previous disc expansion (see Fig. 4). The outburst amplitude is then very reduced.

As noted by King \& Cannizzo (1998), the light curves of dwarf novae do not show such drastic variations of the outburst peak luminosity. Contrary to Schreiber et al. (2000), our results exclude drastic variations of mass transfer rate such as those observed in AM Her.

\section{Conclusion}

Since the work of Meyer \& Meyer-Hofmeister (1983) it has been believed that the $\mathrm{Z}$ Cam behaviour is due to mass transfer rate enhancements that lead to phases of the outburst cycle during which the accretion disc is steady (standstills). However, actual models produced light-curve whose properties did not really correspond to observations (Lin et al. 1985; King \& Cannizzo 1998). In particular, it was not easy to reproduce the correct magnitude difference $\left(\Delta_{\text {mag }} \simeq 0.7 \mathrm{mag}\right)$ between the standstills and the outburst peaks and impossible to obtain inside-out outburst with only moderate mass-transfer variations (Smak 1996). We show that one can account for the observed $\Delta_{\text {mag }}$ if the amplitude of the mass transfer variations are not too large (typically factors 2 or less), as suggested by Lin et al. (1985). An even better agreement is obtained when one takes into account the energy released by the mass-transfer stream impact onto the disc and by tidal 
torque dissipation. Then the required mass transfer rates are also in much better agreement with the expectations for these systems. The sequence of long and short outbursts, the possibility to obtain inside-out outbursts and the characteristic time-scales of the light curves are also in very good agreement with observations.

There are no reasons to believe that mass-transfer rate variations would be restricted to some particular subclasses of dwarf-nova systems. On the contrary, the large variety of outburst shapes (duration and type, outsidein and inside-out) observed in the light curves can be explained by these fluctuations (Hameury et al. 2000; Paper I). Schreiber et al. (2000) argued that mass-transfer rate variations similar to those observed in AM Her should also be present in dwarf novae. However, if one uses correct outer boundary conditions, one obtains mini-outbursts fainter by about 2 mag during what would correspond to low states in AM Her systems. This has not been observed in dwarf novae; moreover, significant drops of the mass transfer rate should result in corresponding drops of the hot spot luminosity, which have not been observed either. In fact, in the few cases where mass-transfer rate variations in dwarf-nova systems seem to be observed these variations are order of magnitudes lower.

Similarly, if the mass transfer rate of nova-like systems were to undergo variations similar to those observed in AM Her systems, most if not all nova-like stars should exhibit a dwarf-nova behaviour, which obviously is not the case. It is interesting to note that we know about 30 Z Cam systems, among several hundred CVs; the catalogue of Ritter \& Kolb (1998) lists 16 Z Cam stars with known orbital periods, among a total of approximately 140 systems in the same orbital period range, meaning that in the Z Cam period range, there is one chance out of 9 for a system to cross the stability limit, indicating that the mass-transfer rate varies by a few tens of percent.

The origin of these variations is not yet understood. It is known (Smak 1995, 1996) that during dwarf-nova outbursts, there is a significant enhancement of the mass transfer rate; but this could not account for erratic variations observed sometimes in quiescence. Stellar spots are a good candidate for explaining these. Whatever the mechanism, it should be influenced by the nature of the primary and/or the evolutionary status of the secondary since we have shown that the pronounced low states of the type observed in magnetic systems and in VY Scl cannot exist in most of dwarf novae.
Acknowledgements. The authors wish to thank the referee, Prof. Y. Osaki, for his very interesting comments and inspiring questions about the outburst types of Z Cam systems. This work was supported in part by a grant from Programme National de Physique Stellaire of the CNRS.

\section{References}

Baraffe, I., \& Kolb, U. 2000, MNRAS, 318, 354

Buat-Ménard, V., Hameury, J.-M., \& Lasota, J.-P. 2001, A\&A, 366, 612 (Paper I)

Esin, A. A., Lasota, J.-P., \& Hynes, R. I. 2000, A\&A 354, 987

Garnavich, P., \& Szkody, P. 1988, PASP, 100, 1522

Gontikakis, C., \& Hameury, J.-M. 1993, A\&A, 271, 118

Hameury, J.-M., Lasota, J.-P., \& Warner, B. 2000, A\&A, 353, 244

Hameury, J.-M., Menou, K., Dubus, G., Lasota, J.-P., \& Huré, J.-M. 1998, MNRAS, 298, 1048

Kalużny, J. 1986, IAUC, 2487

Kato, T. 1999, IBVS, 4678

King, A. R., \& Cannizzo, J. K. 1998, ApJ, 499, 348

Lasota, J.-P. 2000, NAR, in press

Lin, D. N. C., Papaloizou, J. C. B., \& Faulkner, J. 1985, MNRAS, 212, 105

Lubow, S. H., \& Shu, F. H. 1975, ApJ, 198, 383

Livio, M., \& Pringle, J. 1994, ApJ, 427, 956

Meyer, F., \& Meyer-Hofmeister, E. 1983, A\&A, 121, 29

Oppenheimer, B. D., Kenyon, S. J., \& Mattei, J. A. 1998, AJ, 115,1175

Osaki, Y. 1974, PASJ, 26, 429

Paczyński, B. 1977, ApJ, 216, 822

Ringwald, F. A., Thortensen, J. R., Honeycutt, R. K., \& Smith, R. C. 1996, AJ, 111, 2077

Ritter, H., \& Kolb, U. 1998, A\&AS, 129, 83 Z

Robertson, J. W., \& Honeycutt, R. K. 1996, AJ, 112, 2248

Schreiber, M. R., Gänsicke, B. T., \& Hessman, F. V. 2000, A\&A, 358, 221

Smak, J. 1983, ApJ, 272, 234

Smak, J. 1995, Acta Astr., 45, 355

Smak, J. 1996, in Cataclysmic Variables and Related Objects, eds. A. Evans, \& J. H. Woods (Kluwer, Dordrecht), 45

Smak, J. 1998, Acta Astr., 48, 677

Smak, J. 1999, Acta Astr., 49, 383

Smak, J. 2000, NAR, 44, 171

Stehle, R., King, A. R., \& Rudge, C. 2001, MNRAS, in press [astro-ph/0012379]

Szkody, P., \& Mattei, J. A. 1984, PASP, 96, 988

Warner, B. 1995, Cataclysmic Variable Stars (Cambridge University Press, Cambridge)

Wood, J. H., Naylor, T., Hassal, B. J. M., \& Ramseyer, T. F. 1995, MNRAS, 273, 772 